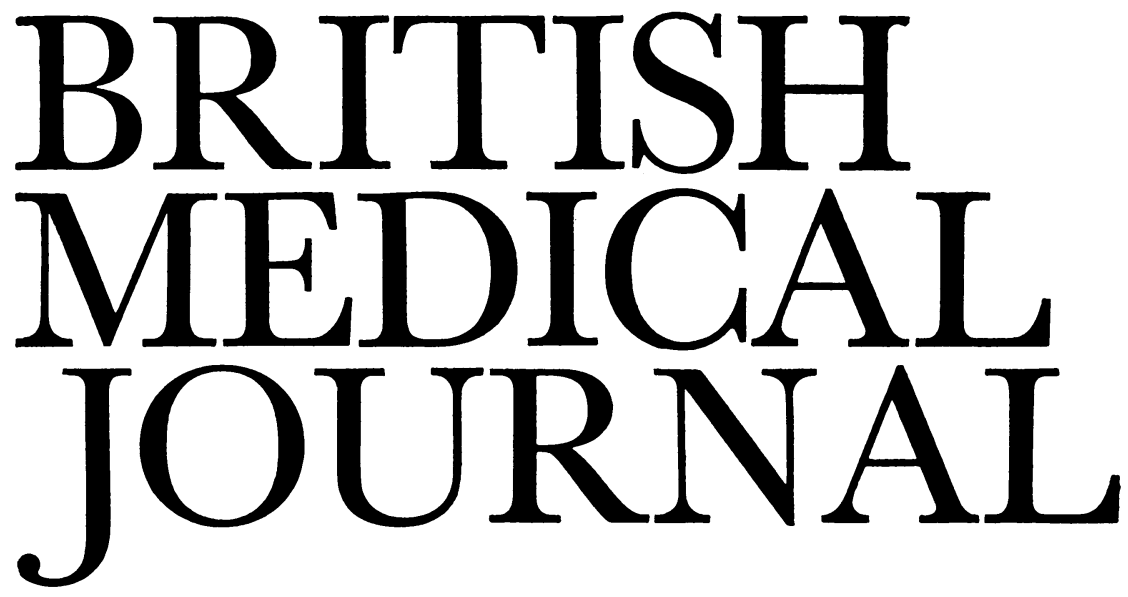

LONDON, SATURDAY 28 MARCH 1981

\title{
Treatment of anaphylactic shock
}

Anaphylactic shock is a relatively uncommon emergency, but it requires prompt and vigorous treatment. Anaphylaxis may occur after foods, including nuts, shellfish, eggs, and milk, and after envenomation by insects and snakes. ${ }^{1}$ In clinical practice anaphylaxis is encountered most often after treatment with certain drugs, ${ }^{2-4}$ including antibiotics (especially penicillins, cephalosporins, streptomycin), non-steroidal anti-inflammatory agents, cardiac depressant antiarrhythmics (procainamide and quinidine), heparin, parenteral iron, neuromuscular blocking agents, polypeptides, heterologous sera and blood products, vaccines, and allergenic extracts given for desensitisation. Atopic individuals are especially susceptible, and reactions are more likely to occur after parenteral than oral administration. Almost always the patient gives a history of previous exposure to the drug. The incidence of anaphylactic reactions in the population is unknown, but they have been reported in $0.06 \%$ of patients in hospital. ${ }^{5}$

The severe manifestations of anaphylaxis include a sudden fall in blood pressure due to vasodilatation of the arterioles and venules as well as to increased capillary permeability, angiooedema (including laryngeal oedema), and bronchospasm. Milder signs and symptoms include rhinitis, pruritus, urticaria, vomiting, diarrhoea, and abdominal pain. The fall in cardiac output which accompanies a reduced venous return of blood to the heart may cause secondary myocardial and tissue hypoxia, with cardiac arrhythmias and a metabolic acidosis completing a vicious circle.

The keystone of effective treatment is adrenaline: as soon as the reaction is recognised $500-1000 \mu \mathrm{g}(0.5$ to $1.0 \mathrm{ml}$ of socalled 1:1000) should be injected intramuscularly (not subcutaneously because absorption is too slow, especially in the presence of shock) since it may prevent further progression. The beneficial effects of adrenaline are due to its mixed sympathomimetic properties. Its alpha-adrenoceptor agonist activity causes constriction of the peripheral vasculature, while its beta-adrenoceptor agonist activity is valuable as a bronchodilator. The adrenaline may also impair the further release of chemical mediators concerned in the pathogenesis of the reaction. Intramuscular injections of adrenaline should be repeated every 15 minutes until improvement occurs.

Though histamine is only one of the mediators concerned in anaphylaxis it may be the most important. Increased blood concentrations of histamine have been described during anaphylaxis and appear to correlate with the degree of hypotension. ${ }^{6}$ A slow intravenous injection of an $\mathrm{H}_{1}$ antagonist (such as $10-20 \mathrm{mg}$ chlorpheniramine) should be given immediately after intramuscular adrenaline and repeated over the subsequent 24 to 48 hours to prevent relapse. $\mathrm{H}_{1}$-antagonists are particularly effective in the management of angio-oedema, pruritus, and urticaria, but remain second-line treatment.

Application of a tourniquet proximal to the site of injection of the antigen may prevent the systemic absorption of chemical mediators. Unfortunately, most parenteral drugs are injected into the buttock or upper deltoid region, making the application of an effective tourniquet impossible. Local injection of adrenaline into the site of antigen administration is beneficial only in the early stages of the reaction.

Intravenous corticosteroids (such as $200 \mathrm{mg}$ hydrocortisone hemisuccinate) have little place in the immediate management of anaphylaxis, since their beneficial effects are delayed for several hours. In severely ill patients, however, early administration may help prevent deterioration after the primary treatment has been given.

Most patients with anaphylaxis respond to the combination of intramuscular adrenaline and an intravenous $\mathrm{H}_{1}$-antagonist. Continuing deterioration with circulatory collapse, bronchospasm, or laryngeal oedema requires further treatment. Circulatory collapse requires volume replacement by intravenous fluids monitored, if possible, by a central venous pressure line. If electrolyte solutions are used large volumes may be necessary because in severe anaphylactic shock the plasma loss may constitute $20-40 \%$ of the plasma volume. ${ }^{6}$ Colloid solutions such as plasma protein fraction or dextran are theoretically preferable but may themselves release histamine, though in severe anaphylaxis intracellular stores of histamine are likely to have been depleted. Bronchospasm refractory to intramuscular adrenaline requires treatment with intravenous aminophylline, a nebulised beta ${ }_{2}$-agonist (such as salbutamol or terbutaline), oxygen, and (if necessary) assisted ventilation. Respiratory obstruction due to laryngeal oedema may require emergency tracheostomy since it is often impossible to insert an endotracheal tube.

Effective treatment of anaphylactic shock clearly depends on the severity of the clinical condition and the circumstances under which it occurs. Since, however, treatment with intramuscular adrenaline is relatively simple and effective, all 
doctors, community nurses, ${ }^{7}$ and medical auxiliaries, such as chiropodists, who might encounter this emergency should carry a supply (which needs to be renewed every six months) and be fully instructed in its use.

${ }^{1}$ Patterson R. Allergic diseases: diagnosis and management. Philadelphia: J B Lippincott, 1972.

${ }^{2}$ Dukes MNG, ed. Meyler's side effects of drugs. Vol 8. Amsterdam: Excerpta Medica, 1975.

3 Dukes MNG, ed. Side effects of drugs. Annual 1. Amsterdam: Excerpta Medica, 1977.

4 Dukes MNG, ed. Side effects of drugs. Annual 2. Amsterdam: Excerpta Medica, 1978.

${ }^{5}$ Boston Collaborative Drug Surveillance Program. Drug-induced anaphylaxis. A cooperative study. $\mathscr{F} A M A$ 1973;224:613-5.

${ }^{6}$ Fisher $M$. Blood volume replacement in acute anaphylactic cardiovascular collapse related to anaesthesia. Brf Anaesth 1977;49:1023-6.

${ }^{7}$ Anonymous. Anaphylaxis and the community nurse. Adverse Drug Reaction Bulletin 1977; No $65: 228-31$.

\section{Bladder cancer}

About 8000 new patients are seen each year ${ }^{1}$ in Britain with tumours of the urothelium. Their management forms a substantial part of the work of a urological department, especially as neither cytological examination of the urine nor any other tumour marker can substitute for the time-honoured "check cystoscopy."

Two-thirds of the patients have superficial papillary tumours when first seen, but two-thirds of these will have a recurrence, and $10-20 \%$ of these recurrences will have progressed to a higher grade. ${ }^{2}$ Overall, about $60 \%$ of patients with superficial tumours and $25 \%$ of patients with invasive tumours are alive five years later. Progress in the management of these tumours has been disappointing, with little change in the past 40 years in the survival of patients with superficial tumours. The fiveyear survival rate for invasive tumours has improved, but at least in part as a result of reduced postoperative mortality. ${ }^{3}$

Endoscopic cystodiathermy or transurethral resection remains the initial treatment for superficial tumours, but with such a high recurrence rate these tumours ought to be suitable for intravesical chemotherapy. For intravesical instillation, thiotepa, ethoglucid, doxorubicin, bleomycin, and mitomycin $\mathrm{C}$ all seem effective agents for these tumours. One study of the effect of a single dose of thiotepa given immediately after endoscopic treatment of superficial tumours showed a substantial reduction in the incidence of tumour recurrence in treated patients compared with the controls. ${ }^{4}$ Further clinical trials are in progress, but difficulties have arisen in defining indications, timing of treatment, and criteria of response, while another problem has been the inconvenience of repeated intravesical instillations. A drug given by mouth and active when excreted in the urine would be a much more attractive approach to this form of treatment, and methotrexate has shown some initial success. ${ }^{5}$

A valuable recent aid to assessing bladder tumours has been the routine use of small "pinch" biopsy specimens taken from apparently normal or red and "suspicious" mucosa at the time of the initial assessment of the tumour. ${ }^{6}$ In one-third of the biopsy specimens taken from normal mucosa there was a urothelial abnormality, and $4.5 \%$ of these were carcinomas; half of the samples from flat, red mucosa were normal, and $14 \%$ of these were carcinomas; where the mucosa was red and raised (or mossy) $42 \%$ showed carcinoma and only $14 \%$ were normal. ${ }^{7}$ These results show how common is widespread mucosal change in bladder cancer; the long-term follow-up results of patients undergoing multiple mucosal biopsies may help to define those requiring earlier or possibly different initial treatment.

The management of a patient whose tumour has started to invade the bladder wall consists in endoscopic resection when the tumour is relatively small and superficial and a combination of radiotherapy and cystectomy when it has invaded deeply into the bladder muscle. In practice this approach is full of doubts, uncertainties, and unresolved questions. One problem is how to establish the depth of invasion by the tumour. Clinical bimanual examination has a wide margin of error, usually considerably understaging the tumour, while judging the depth of penetration from transurethral resection or biopsy samples is incomplete unless the full thickness of the bladder wall can be studied. Possibly all invasive tumours should be considered together with more emphasis being placed on the histological grade. ${ }^{8}$

The next problem has been to determine the optimum combination of radiotherapy and cystectomy from among choices including low-dose $(20 \mathrm{~Gy})$ radiotherapy and immediate cystectomy; radiotherapy ( $40 \mathrm{~Gy}$ ) followed by review and removal of only those bladders where the stage of the tumour is less than originally thought: radiotherapy (40 Gy) followed by cystectomy if the patient is under the age of 65 ; and a full radical course of radiotherapy for all cases with review at six months, removing those bladders with persistent or recurrent tumour"salvaging" the patient. This plethora of choices will continue until comparative data are available-and the prospects for this are not encouraging. Moreover, any differences between these choices now seem less important than the disturbingly high incidence of both local and distant metastases whatever approach is used. ${ }^{9}$ Even the most ardent supporter of either radical radiotherapy or radical cystectomy must admit that when recurrences develop the object of the radical treatment has failed-and the five-year failure rate is around $75 \%$ for invasive (T3) tumours.

The root cause of this failure is our inability to screen for micrometastases. All who carry out radical measures to cure cancer, and a cystourethrectomy must be among the more radical, have to rely on the best available screening methods. The reported recurrence rates for bladder cancer ${ }^{9}$ show very clearly that the available methods are letting us down and that a different approach, rather than a variation on the same approach, is now necessary. At present the only feasible contender is chemotherapy, but so far few drugs have shown any worthwhile effect against bladder cancer. ${ }^{10}$ Both methotrexate and cis-platinum have given encouraging response rates, and already several groups have incorporated these agents into co-operative clinical trials. Nevertheless, our range of drugs is still very limited, and more phase I and phase II studies are needed to find safe, acceptable agents. Before taking on the difficult task of co-operative trials urologists will want good prospects of a worthwhile conclusion, so that they will welcome the cu-ordination of these efforts by the Medical Research Council.

1 Tate HC, Rawlinson JB, Freedman LS. Randomised comparative studies in the treatment of cancer in the United Kingdom: room for improvement? Lancet 1979 ;ii:623-5.

2 Gilbert HA, Logan JL, Kagan AR, et al. The natural history of papillary transitional cell carcinoma of the bladder and its treatment in an unselected population on the basis of histologic grading. 7 Urol 1978; 119:488-92.

${ }^{3}$ McCarron JP, Marshall VF. The survival of patients with bladder tumors treated by surgery: comparative results of an old and a recent series. $\mathcal{f}$ Urol $1979 ; 122: 322-4$. 